\title{
The Efficacy and Safety of Surgical Treatment for Patients With Tuberculosis Destroyed Lung With or Without Chronic Pulmonary Aspergillosis
}

\author{
Hongyun Ruan ${ }^{1}$ Changfan Gong ${ }^{2} \cdot J^{\text {Jinxiang Wang }}{ }^{3}$
}

Accepted: 11 January 2021/Published online: 8 February 2021

(C) The Author(s) 2021

\begin{abstract}
Background To evaluate the efficacy and safety of surgical treatment of tuberculosis destroyed lung (TDL), and the influence of chronic pulmonary aspergillosis (CPA) on the outcomes of surgical treatment of TDL.

Methods We performed a retrospective analysis of 113 patients with TDL who underwent surgical treatment from January 2005 to December 2019. Among them, 30 of these cases were complicated with CPA. The patients were divided into two groups: TDL group and TDL + CPA group. We analyzed the effectiveness and safety of surgical treatment of TDL, and further compared the effectiveness and safety of surgical treatment of TDL with or withoutthe presence of CPA.

Results The TDL + CPA group had a significantly higher age $(P=0.003)$, symptoms of hemoptysis $(P=0.000)$, and a higher proportion of patients with preoperative serum albumin $<30 \mathrm{~g} / \mathrm{L}(P=0.014)$ as compared with TDL group. For all enrolled patients, the incidence of severe postoperative complications was $12.4 \%$ (14/113) and the postoperative mortality within 30 days after discharge was $4.4 \%(5 / 113) .86 .7 \%$ (98/113) of the patients recovered and discharged, the incidence of severe postoperative complications in the TDA + CPA group was higher than that of TDL group (23.3\% vs $8.4 \%, P=0.034)$, although there was no difference in mortality between the two groups $(P=1.000)$. A binary logistic regression analysis showed that the independent risk factors for severe postoperative complications were male (OR $25.24,95 \%$ CI 2.31-275.64; $P=0.008$ ) and age $\geq 40$ years (OR 10.34, 95\% CI 1.56-68.65; $P=0.016$ ).

Conclusion Surgical treatment for patients with TDL is effective with an acceptable mortality rate whether or not the disease is complicated with CPA. The independent risk factors identified for severe postoperative complications in patients with TDL were male and $\geq 40$ years old. It implies that when treating patients with TDA + CPA, particular attention should be paid to these patients who have these independent risk factors to avoid a poor outcome.
\end{abstract}

Jinxiang Wang

wangjx0090@sina.com

Hongyun Ruan

13661154466@126.com

Changfan Gong

wawdhne@163.com

1 Cardiopulmonary Function Department, Beijing Chest

Hospital, Capital Medical University, Beijing, China
Department of Thoracic Surgery, Beijing Chest Hospital, Capital Medical University, Beijing, China

3 Department of Pulmonary and Critical Care Medicine, Beijing Luhe Hospital, Capital Medical University, Beijing, China 


\section{Introduction}

Chronic lung infection can result in destroyed lung, characterized by diffuse lung structural damage, is more common in developing countries. Tuberculosis is the most common cause of destroyed lung [1,2]. The prevalence of CPA ranged from 6.3 to $13.7 \%$ in patients previously or currently being treated for tuberculosis [3], residual cavity is more susceptible to complications of chronic pulmonary as per gillosis (CPA) [4, 5]. The most common symptom of pulmonary tuberculosis and CPA is hemoptysis [6-9], and massive hemoptysis is an indication of surgical treatment [2, 10-12]. Surgical treatment is also a necessary and important means for treating patients with multidrug resistant tuberculosis (MDR-TB) [11, 13, 14] or poor response to antifungal therapy [15]. Many studies have shown that the surgical treatment of tuberculosis destroyed lung (TDL) or CPA is effective, with acceptable postoperative complications and mortality. Therefore, surgical treatment has become an important treatment option for this population [6, 7, 9, 16-19].

The main purpose of surgical treatment of TDL is to solve life-threatening complications and improve the quality of life [20]. Surgical treatment can also help treat MDR-TB and prevent recurrence of the disease [11,13]. Antifungal therapy is the main treatment of CPA [15]. However, antifungal therapy requires a long course and has a low success rate [21]. If there is life-threatening hemoptysis, or a poor response to antifungal therapy, surgical treatment should be considered [15]. Moreover, surgical resection may be a better option than long-term antifungal therapy if patients cannot afford the cost of long-term treatment, drug treatment failure or intolerance to long-term oral antifungal therapy $[15,22]$. If patients have both TDL and CPA, the treatment is even more complicated. There have been few studies in this area, but one study involving some CPA patients complicated with TBL reported that surgical treatment can benefit patients with CPA [7].

In order to evaluate the efficacy and safety of surgical treatment of TDLwith or without CPA, we retrospectively analyzed 113 patients who underwent surgical treatment for TDL in Beijing Chest Hospital from January 2005 to December 2019; thirty of the included cases were complicated with CPA.

\section{Materials and methods}

\section{Study design and objectives}

From January 2005 to December 2019, all patients with TDL who underwent surgical treatment in Beijing Chest Hospital were enrolled in this study. Among them, 30 patients were confirmed as Aspergillus infection by histopathology. This study was approved by the Ethics Committee of Beijing Chest Hospital affiliated to Capital Medical University (Ethics number: clinical study 2018 (43)). The requirement for informed consent from the patients was waived by the ethical review board.

The patients were divided into two groups: TDL group and TDL + CPA group. General clinical data were collected, including gender, age, smoking status, alcohol abuse, body mass index, comorbidities, symptoms (hemoptysis, cough, fever, dyspnea, and chest pain), preoperative lung function, routine laboratory examination, CPA classification, intraoperative blood loss, severe postoperative complications, death (postoperative during hospitalization and within 30 days after discharge), and the cause of death. Severe postoperative complications included severe respiratory failure due to infection requiring invasive mechanical ventilation for more than $72 \mathrm{~h}$, heart failure, empyema, bronchopleural fistula, and reoperation due to massive postoperative bleeding.

The diagnosis of CPA was based on the criteria of ERS and ESCMID [22]: one or more cavities with or without a fungal ball present or nodules on thoracic imaging; direct evidence of Aspergillus infection (microscopy or culture from biopsy) or, an immunological response to Aspergillus spp. and exclusion of alternative diagnoses, all present for at least 3 months. The diagnosis of CPA included simple aspergilloma, chronic cavitary pulmonary aspergillosis (CCPA), chronic fibrosing pulmonary aspergillosis (CFPA), Aspergillusnodule and subacute invasive aspergillosis (SAIA).

Jaeger Master Screen PFT was used to measure lung function. Lung diffusion capacity for carbon monoxide was measured using single breath method. Lung function data include: forced expiratory volume in one second of predicted $\left(\mathrm{FEV}_{1} \%\right.$ pred); forced vital capacity of predicted (FVC\% pred); the ratio of forced expiratory volume in one second to forced vital capacity $\left(\mathrm{FEV}_{1} / \mathrm{FVC}\right)$; vital capacity of predicted ( $\mathrm{VC} \%$ pred); maximal minute ventilation of predicted (MMV\% pred); inspiratory capacity of predicted (IC\% pred); functional residual volume of predicted (FRC\% pred); residual volume of predicted (RV\% pred); total lung capacity of predicted (TLC\% pred); the ratio of total lung capacity to residual volume (RV/TLC); and lung diffusion capacity for carbon monoxide of predicted $\left(\mathrm{D}_{\mathrm{L}}\right.$ $\mathrm{CO} \%$ pred).

The surgical indication of TDL was that the lesions were confined to the ipsilateral lung with the following conditions were met: TDL and CPA were located in the ipsilateral lung; massive hemoptysis; persistent positive of sputum smear and/or culture of Mycobacterium tuberculosis; antituberculosis treatment was ineffective or intolerable; recurrent infection of TDL. Surgical 
contraindications were as follows: respiratory failure in preoperative blood gas analysis; serious comorbidities; bilateral involvement of lesions. Whether or not to take surgical treatment for TDL patients was discussed and decided by a team of experts from radiology, thoracic surgery and anesthesiology.

The aim of our study was to evaluate the efficacy and safety of surgical treatment of TDL and by comparative analysis the efficacy and safety of surgical treatment of TDL complicated with CPA.

\section{Statistical analysis}

SPSS version 21.0 for Windows software (SPSS Inc.,Chicago, IL, USA) was used for data management and statistical analysis. Continuous variables data were expressed as the mean $\pm \mathrm{SD}$, whereas categorical data were presented as a number or percentage. Continuous variables data were compared using the parametric unpaired, twoindependent-group Student's t-test. Binary logistic regression analysis was used to assess the following risk factorsfor severe postoperative complications: gender, age $\geq 40$ years [23], smoking, comorbidities, CPA, low body mass index $\left(<18.5 \mathrm{~kg} / \mathrm{m}^{2}\right)$, anemia (hemoglobin $<90 \mathrm{~g} / \mathrm{L}$ ) and low serum albumin $(<30 \mathrm{~g} / \mathrm{L})$ before operation. We calculated the risk factors for odds ratio (OR) and $95 \%$ for confidence interval (CI). The Pearson Chi-square test or continuous correction Chi-square test was used to compare the counting data and $P<0.05$ was considered statistically significant.

\section{Result}

A total of 113 patients with TDL who underwent surgical treatment were enrolled, 51 males and 62 females, with a mean age of $39.2 \pm 13.6$ years. Thirty patients had TDL complicated with CPA, 17 males and 13 females with a mean age of $46.2 \pm 15.3$ years. Four cases in CPA group and nine cases in TDL + CPA group did not receive antituberculosis therapy before operation. For 30 patients of TDL complicated with CPA, three cases received itraconazole and 27 cases received voriconazole for antifungal treatment during perioperative period. The distribution of CPA types included, nine cases of CCPA, two cases of CFPA, three cases of simple aspergilloma, five cases of Aspergillus nodule and four cases of SAIA. In the TDL group, three cases were complicated with nontuberculous mycobacterium infection (Table 1).

We found the mean age was higher $(46.2 \pm 15.3$ years vs. $36.7 \pm 12.0, P=0.003)$, and the symptom of hemoptysis was more common $(P=0.000)$ in TDL + CPA group as compared to the TDL group; other symptoms of cough, expectoration, fever and dyspnea had no observable differences between the two groups. We also observed that $\mathrm{FEV}_{1} \%$ pred was higher in the TDL $+\mathrm{CPA}$ group than in the TDL group $(67.8 \pm 24.3$ vs. $54.2 \pm 15.1, P=0.035)$, but no statistical difference $(P>0.05)$ between the two groups in $\mathrm{FVC} \%$ pred, $\mathrm{FEV}_{1} / \mathrm{FVC}, \mathrm{MMV} \%$ pred, $\mathrm{VC} \%$ pred, IC\% pred, RV\% pred, RV/TLC, TLC\% pred and $\mathrm{D}_{\mathrm{L}} \mathrm{CO} \%$ pred (Table 1).

The proportion of patients with preoperative serum albumin $<30 \mathrm{~g} / \mathrm{L}$ in the TDL + CPA group was higher than that in the TDL group $(P=0.014)$. There was no statistical difference $(P>0.05)$ between the two groups for body mass index, smoking status, main comorbidities, preoperative anemia (hemoglobin $<90 \mathrm{~g} / \mathrm{L}$ ), preoperative arterial blood gas analysis index and the incidence of MDR-TB. Three cases of nontuberculous mycobacterium infection in the TDL group (Table 1).

Among 113 patients, 34 patients underwent lobectomy and 79 underwent pneumonectomy. There was no recurrence of massive hemoptysis, the incidence of severe postoperative complications was $12.4 \%$ (14/113), and the postoperative mortality within 30 days after discharge was $4.4 \%(5 / 113), 86.7 \%(98 / 113)$ of the patients recovered gradually and discharged. The incidence of severe postoperative complications in TDL + CPA group was significantly higher than that in the TDL group $(23.3 \%$ vs. $8.4 \%, P=0.034)$, although there was nodifference in the amount of intraoperative blood loss, the postoperative mortality within 30 days after discharge, and the postoperative length of hospital stay (Table 2).

In the TDL group, seven cases had severe postoperative complications, including four cases of massive postoperative bleeding, one case of heart failure, two cases of severe respiratory failure due to recurrent pulmonary infection. Four cases died postoperative or within 30 days following discharge, one case died of severe respiratory failure and three cases died of massive hemorrhage (Table 2).

In TDL + CPA group, seven cases had severe postoperative complications, including one case of empyema, one case of massive postoperative bleeding, four cases of severe respiratory failure, and 1 case of bronchopleural fistula. One patient died of bronchopleural fistula complications (Table 2).

Reoperation for severe postoperative complications: In TDL group, one patient with massive postoperative bleeding survived after reoperation, and the other three patients with massive postoperative bleeding were critically ill and did not have the conditions for reoperation and died. In TDL + CPA group, the patients with massive postoperative bleeding or bronchopleural fistula were reoperated, and the patient with bronchopleural fistula died.

The binary logistic regression analysis showed that the independent risk factors for severe postoperative 
Table 1 Baseline clinical characteristics of TDL with or without CPA

\begin{tabular}{|c|c|c|c|}
\hline Characteristics & TDL group $(n=83)$ & TDL + CPA group $(n=30)$ & $P$ Value \\
\hline \multicolumn{4}{|l|}{ Sex } \\
\hline Male & $34(41.0)$ & $17(56.7)$ & 0.139 \\
\hline Age & $36.7 \pm 12.0$ & $46.2 \pm 15.3$ & 0.003 \\
\hline $\mathrm{BMI}<18.5 \mathrm{~kg} / \mathrm{m}^{2}$ & $13(15.7)$ & $7(23.3)$ & 0.346 \\
\hline Smoking status: Smoking & $11(13.3)$ & $5(16.7)$ & 0.646 \\
\hline Comorbidities* & $6(7.2)$ & $3(10.0)$ & 0.931 \\
\hline \multicolumn{4}{|l|}{ Symptom } \\
\hline Hemoptysis & $20(24.1)$ & $19(63.3)$ & 0.000 \\
\hline Cough & $39(47.0)$ & $11(36.7)$ & 0.329 \\
\hline Fever & $7(8.4)$ & $4(13.3)$ & 0.677 \\
\hline Dyspnea & $28(33.7)$ & $5(16.7)$ & 0.078 \\
\hline Hemoglobin $<90 \mathrm{~g} / \mathrm{L}$ & $7(8.4)$ & $3(10.0)$ & 1.000 \\
\hline Serumalbumin $<30 \mathrm{~g} / \mathrm{L}$ & $3(3.6)$ & $6(20.0)$ & 0.014 \\
\hline MDR-TB & $9(10.8)$ & $4(13.3)$ & 0.974 \\
\hline \multicolumn{4}{|l|}{ Lung function } \\
\hline $\mathrm{FEV}_{1}(\%$ pred $)$ & $54.2 \pm 15.1$ & $67.8 \pm 24.3$ & 0.035 \\
\hline FVC (\% pred) & $62.9 \pm 15.6$ & $70.7 \pm 22.2$ & 0.173 \\
\hline $\mathrm{FEV}_{1} / \mathrm{FVC}$ & $74.0 \pm 12.5$ & $79.1 \pm 13.8$ & 0.139 \\
\hline $\mathrm{RV}(\%$ pred $)$ & $123.1 \pm 37.2$ & $115.8 \pm 38.8$ & 0.470 \\
\hline TLC (\% pred) & $79.8 \pm 17.6$ & $84.5 \pm 17.0$ & 0.311 \\
\hline RV/TLC & $150.9 \pm 28.1$ & $132.3 \pm 41.7$ & 0.088 \\
\hline IC (\% pred $)$ & $67.9 \pm 27.2$ & $64.6 \pm 26.5$ & 0.643 \\
\hline $\mathrm{VC}(\%$ pred $)$ & $61.5 \pm 15.2$ & $70.2 \pm 20.9$ & 0.110 \\
\hline MMV (\% pred) & $53.7 \pm 14.7$ & $66.6 \pm 28.4$ & 0.078 \\
\hline $\mathrm{D}_{\mathrm{L}} \mathrm{CO}(\%$ pred $)$ & $58.5 \pm 15.7$ & $74.4 \pm 34.2$ & 0.070 \\
\hline \multicolumn{4}{|l|}{ Arterial blood gas analysis } \\
\hline $\mathrm{pH}$ value & $7.40 \pm 0.04$ & $7.41 \pm 0.03$ & 0.650 \\
\hline $\mathrm{PaCO}_{2}$ & $42.8 \pm 4.0$ & $43.4 \pm 3.8$ & 0.556 \\
\hline $\mathrm{PaO}_{2}$ & $104.6 \pm 37.8$ & $101.9 \pm 28.2$ & 0.810 \\
\hline
\end{tabular}

Data are presented as mean \pm SD or number $(\%)$

$T D L$ tuberculosis destroyed lung; $C P A$ chronic pulmonary aspergillosis; $F E V_{1}$ (\% pred), forced expiratory volume in one second of predicted; $F V C$ (\% pred) forced vital capacity of predicted; $F E V_{l} / F V C$ the ratio of forced expiratory volume in one second to forced vital capacity; $R V(\%$ pred), residual volume of predicted; $T L C$ (\% pred), total lung capacity of predicted; $R V / T L C$ the ratio of total lung capacity to residual volume; $I C$ (\% pred), inspiratory capacity of predicted; $V C$ (\% pred), vital capacity of predicted; $M M V(\%$ pred), maximal minute ventilation of predicted; $D_{L} C O$ (\% pred), lung diffusion capacity of predicted

*Comorbidities in TDL group included 4 cases of diabetes, 1 case of congenital atrial septal defect and 1 case of ankylosing spondylitis; comorbidities in TDL + CPA group included 1 case of hypertension and 2 cases of diabetes

complications were male gender (OR 25.24, 95\% CI 2.31-275.64; $P=0.008)$ and age $\geq 40$ years (OR 10.34, 95\% CI 1.56-68.65; $P=0.016$ ) (Table 3).

\section{Discussion}

Destroyed lung is an irreversible disease defined by radiology with the physiological characteristic of decreased ventilation/perfusion ratio, with the primary cause from pulmonary tuberculosis [2], that is common in countries with underdeveloped health systems [24]. In Asia and Africa, the co-infection rate of Aspergillus and tuberculosis is $15.4 \%$, and it is higher in individuals above 40 years old [25]. Male tuberculosis patients are more likely to be infected with fungi, which may be related to high exposure to the surrounding environment [26]. In our study, the rate of dyspnea had a trend higher in TDL group than TDL + CPA group before surgery $(P=0.078)$, and the blood gas results of the two groups were similar, which may be due to 
Table 2 Clinical outcomes of TDL with or without CPA

\begin{tabular}{|c|c|c|c|}
\hline Characteristics & TDL group $(n=83)$ & TDL + CPA group $(n=30)$ & $P$ Value \\
\hline Intraoperative blood loss, $\mathrm{ml}$ & $871 \pm 796$ & $1393 \pm 1575$ & 0.091 \\
\hline Severe postoperative complications & $7(8.4)$ & $7(23.3)$ & 0.034 \\
\hline Severe respiratory failure & 2 & 4 & \\
\hline Heart failure & 1 & 0 & \\
\hline Empyema & 0 & 1 & \\
\hline Bronchopleural fistula & 0 & 1 & \\
\hline Massive postoperativebleeding & 4 & 1 & \\
\hline Postoperative hospital stay, days & $29.5 \pm 22.1$ & $37.5 \pm 25.5$ & 0.106 \\
\hline Death after operation, during hospitalization or within 30 days of discharge & $4(4.8)$ & $1(3.3)$ & 1.000 \\
\hline
\end{tabular}

Data are presented as mean \pm SD or number $(\%)$

$T D L$ tuberculosis destroyed lung; $C P A$ chronic pulmonary aspergillosis

Table 3 The Binary logistic regression analysis for risk factors of severe postoperative complications in patients with TDL

\begin{tabular}{lllllllll}
\hline Characteristics & $\begin{array}{l}\geq 40 \text { years } \\
\text { old }\end{array}$ & Male & $\begin{array}{l}\text { BMI }<18.5 \mathrm{~kg} / \\
\mathrm{m}^{2}\end{array}$ & Smoking & $\begin{array}{l}\text { Hemoglobin }<90 \mathrm{~g} / \\
\mathrm{L}\end{array}$ & $\begin{array}{l}\text { Albumin }<30 \mathrm{~g} / \\
\mathrm{L}\end{array}$ & CPA & Comorbidities \\
\hline OR & 10.342 & 25.236 & 6.136 & 0.612 & 7.300 & 0.754 & 2.509 \\
$95 \%$ CI & $1.558-68.654$ & $2.310-275.643$ & $0.856-43.977$ & $0.105-3.636$ & $0.808-65.973$ & $0.068-8.324$ & $0.539-11.671$ & $0.097-11.209$ \\
$P$ Value & 0.016 & 0.008 & 0.071 & 0.585 & 0.077 & 0.818 & 0.241 & 0.972 \\
\hline
\end{tabular}

$B M I$ body mass index; $C P A$ chronic pulmonary aspergillosis

the fact that blood gas analysis mainly reflects respiratory function, which is only one of the important factors affecting dyspnea. There was no gender difference in patients with TDL whether or not complicated with CPA $(P=0.139)$, but the age of patients in the TDL + CPA group was higher than that in the TDL group, indicating that elderly patients with TDL are more likely to have fungal infection, consistent with the conclusion of Hosseini et al. [25].

Surgical treatment of destroyed lung can solve lifethreatening complications and improve the quality of life for affected patients [20]. It has been proven that the postoperative complications and mortality of surgical treatment of patients with TDL or CPA are acceptable $[6,7,9,16-18]$. Indications for surgical treatment of destroyed lung include massive hemoptysis, empyema, secondary fungal infection and respiratory failure that have affected the quality of life [2]. When TDL is complicated with MDR-TB, surgical resection is also helpful for negative conversion of MDR-TB and preventing recurrence $[11,13]$. In our results, the incidence of severe complications and mortality were $12.4 \%$ and $4.4 \%$, respectively, similar to those reported in the literature [2, 17-19]. Although the incidence of severe postoperative complications was higher in the TDL + CPA group than in the TDL group, there was no difference in the mortality between the two groups postoperatively during hospitalization or within
30 days after discharge. Therefore, surgical treatment of TDL complicated with CPA was also effective with an acceptable mortality rate.

Patients with pulmonary tuberculosis are susceptible to $\mathrm{CPA}$, and antifungal drugs are the first choice for the treatment $[15,27]$. However, the course of antifungal therapy is long and the success rate is low [21]. Moreover, many CPA patients live in low- and middle-income countries, where surgical resection may be better than longterm antifungal therapy due to poor economic conditions, potential drug treatment failure and intolerance to longterm oral antifungal therapy $[15,22]$. Our study showed that even if the TDL is complicated with CPA or not, surgical treatment is a safe and effective option. Although for patients with ineffective or intolerable medical treatment, as well as patients with life-threatening hemoptysis, especially for the TDL patients with CPA, surgical treatment is a good choice. However, there were some serious complications and deaths after operation, which should be paid attention to and treated actively.

It has been reported that hypoalbuminemia increases the risk of postoperative complications of TDL [2] and is associated with increased mortality in patients with CPA [28]. Our study showed that the independent risk factors of severe postoperative complications in patients with TDL are male gender and an age $\geq 40$ years old, that may be related to the susceptibility of male and elderly patients to 
CPA. However, we did not find that hypoalbuminemia was associated with an increase in severe postoperative complications.

This study had several limitations. Firstly, for the retrospective study, the follow-up time is short, no follow-up of lung function. Therefore, the recovery of postoperative pulmonary function, whether there is chronic respiratory failure and the need for home oxygen therapy, and the postoperative quality of life are not clear, which need to be further studied. Secondly, due to the small number of cases, especially the small number of CPA cases, we were unable to carry out a subgroup analysis on CPA, nor did we conduct subgroup analysis on specific surgical procedures. Finally, owing to the large time span of included cases, there are differences in medical practice and technologies across this time span that would certainly impact the results. However, this is inevitable in any retrospective study.

In conclusion, surgical resection is effective in the treatment of TDL with an acceptable mortality rate whether or not it is complicated with CPA. The independent risk factors for severe postoperative complications in patients with TDL were male and $\geq 40$ years old. The increased risk of postoperative severe complications in patients with TDL complicated with CPA deserves attention.

\section{Compliance with ethical standards}

Conflict of interest The authors declare that they have no conflict of interest.

Open Access This article is licensed under a Creative Commons Attribution 4.0 International License, which permits use, sharing, adaptation, distribution and reproduction in any medium or format, as long as you give appropriate credit to the original author(s) and the source, provide a link to the Creative Commons licence, and indicate if changes were made. The images or other third party material in this article are included in the article's Creative Commons licence, unless indicated otherwise in a credit line to the material. If material is not included in the article's Creative Commons licence and your intended use is not permitted by statutory regulation or exceeds the permitted use, you will need to obtain permission directly from the copyright holder. To view a copy of this licence, visit http://creativecommons. org/licenses/by/4.0/.

\section{References}

1. Eren S, Eren MN, Balci AE (2003) Pneumonectomy in children for destroyed lung and the long-term consequences. J ThoracCardiovascSurg 126(2):574-581

2. Kosif MA, Bayram S, Kiral H et al (2018) Factors affecting complication rates of pneumonectomy in destroyed lung. Turk GogusKalpDamarCerrahisiDerg 26(2):272-278

3. Bongomin F (2020) Post-tuberculosis chronic pulmonary aspergillosis: An emerging public health concern. PLoSPathog 16(8):e1008742
4. Denning DW, Pleuvry A, Cole DC (2011) Global burden of chronic pulmonary aspergillosis as a sequel to pulmonary tuberculosis. Bull World Health Organ 89(12):864-872

5. Page ID, Byanyima R, Hosmane S et al (2019) Chronic pulmonary aspergillosis commonly complicates treated pulmonary tuberculosis with residual cavitation. EurRespir J. https://doi.org/ 10.1183/13993003.01184-2018

6. Bai L, Hong Z, Gong C, Yan D, Liang Z (2012) Surgical treatment efficacy in 172 cases of tuberculosis-destroyed lungs. Eur $\mathbf{J}$ CardiothoracSurg 41(2):335-340

7. He B, Wan C, Zhou W, Rui Y, Shi Y, Su X (2019) Clinical profile and surgical outcome for different types of chronic pulmonary aspergillosis. Am J Transl Res 11(6):3671-3679

8. Mohapatra B, Sivakumar P, Bhattacharya S, Dutta S (2016) Surgical treatment of pulmonary aspergillosis: A single center experience. Lung India 33(1):9-13

9. Regnard JF, Icard P, Nicolosi M et al (2000) Aspergilloma: a series of 89 surgical cases. Ann ThoracSurg 69(3):898-903

10. Issoufou I, Sani R, Belliraj L et al (2016) Pneumonectomy for tuberculosis destroyed lung: a series of 26 operated cases. Rev PneumolClin 72(5):288-292

11. Yuncu G, Ceylan KC, Sevinc S et al (2006) Functional results of surgical treatment of bronchiectasis in a developing country. Arch Bronconeumol 42(4):183-188

12. Patterson TF, Thompson GR, Denning DW et al (2016) Practice guidelines for the diagnosis and management of aspergillosis: 2016 update by the infectious diseases society of America. Clin Infect Dis 63(4):e1-e60

13 Treasure RL, Seaworth BJ (1995) Current role of surgery in Mycobacterium tuberculosis. Ann ThoracSurg. https://doi.org/10. 1016/0003-4975(95)00145-B

14. Kir A, Tahaoğlu K, Okur E, Hatipoğlu T (1997) Role of surgery in multi-drug-resistant tuberculosis: results of 27 cases. Eur J CardiothoracSurg 12(4):531-534

15. Sehgal IS, Dhooria S, Muthu V, Prasad KT, Agarwal R (2020) An overview of the available treatments for chronic cavitary pulmonary aspergillosis. Expert Rev Respir Med 14(7):715-727

16. Halezeroglu S, Keles M, Uysal A et al (1997) Factors affecting postoperative morbidity and mortality in destroyed lung. Ann ThoracSurg 64(6):1635-1638

17. Huang CL, Zhang W, Ni ZY et al (2015) Efficacy of videoassisted thoracoscopic surgery for 29 patients with tuberculosisdestroyed lung. Int J ClinExp Med 8(10):18391-18398

18. Muniappan A, Tapias LF, Butala P et al (2014) Surgical therapy of pulmonary aspergillomas: a 30-year North American experience. Ann ThoracSurg 97(2):432-438

19. Sayir F, Ocakcioglu I, Şehitoğulları A, Çobanoğlu U (2019) Clinical analysis of pneumonectomy for destroyed lung: a retrospective study of 32 patients. Gen ThoracCardiovascSurg 67(6):530-536

20. Tanaka H, Matsumura A, Okumura M, Iuchi K (2005) Pneumonectomy for unilateral destroyed lung with pulmonary hypertension due to systemic blood flow through broncho-pulmonary shunts. Eur J CardiothoracSurg 28(3):389-393

21. Cadranel J, Philippe B, Hennequin C et al (2012) Voriconazole for chronic pulmonary aspergillosis: a prospective multicenter trial. Eur J ClinMicrobiol Infect Dis 31(11):3231-3239

22. Denning DW, Cadranel J, Beigelman-Aubry C et al (2016) Chronic pulmonary aspergillosis: rationale and clinical guidelines for diagnosis and management. EurRespir J 47(1):45-68

23. Li Y, Hu X, Jiang G, Chen C (2017) Pneumonectomy for Treatment of Destroyed Lung: A Retrospective Study of 137 Patients. ThoracCardiovascSurg 65(7):528-534

24. Stephen T, Thankachen R, Madhu AP, Neelakantan N, Shukla V, Korula RJ (2007) Surgical results in bronchiectasis: analysis of 149 patients. Asian CardiovascThorac Ann 15(4):290-296 
25. Hosseini M, Shakerimoghaddam A, Ghazalibina M, Khaledi A (2020) Aspergillus coinfection among patients with pulmonary tuberculosis in Asia and Africa countries; a systematic review and meta-analysis of cross-sectional studies. MicrobPathog 141:104018

26. Astekar M, Bhatiya PS, Sowmya GV (2016) Prevalence and characterization of opportunistic candidal infections among patients with pulmonary tuberculosis. J Oral MaxillofacPathol 20(2):183-189
27. Maghrabi F, Denning DW (2017) The Management of Chronic Pulmonary Aspergillosis: The UK National Aspergillosis Centre Approach. Curr Fungal Infect Rep 11(4):242-251

28 Lowes D, Al-Shair K, Newton PJ et al (2017) Predictors of mortality in chronic pulmonary aspergillosis. EurRespir J. https:// doi.org/10.1183/13993003.01062-2016

Publisher's Note Springer Nature remains neutral with regard to jurisdictional claims in published maps and institutional affiliations. 